\title{
Study and simulation with VHDL-AMS of the electrical impedance of a piezoelectric ultrasonic transducer
}

\author{
Toufik Merdjana, Abdelhafid Chaabi
}

Hyper Frequency and Semiconductor Laboratory (LHS), University Mentouri Constantine1, Algeria

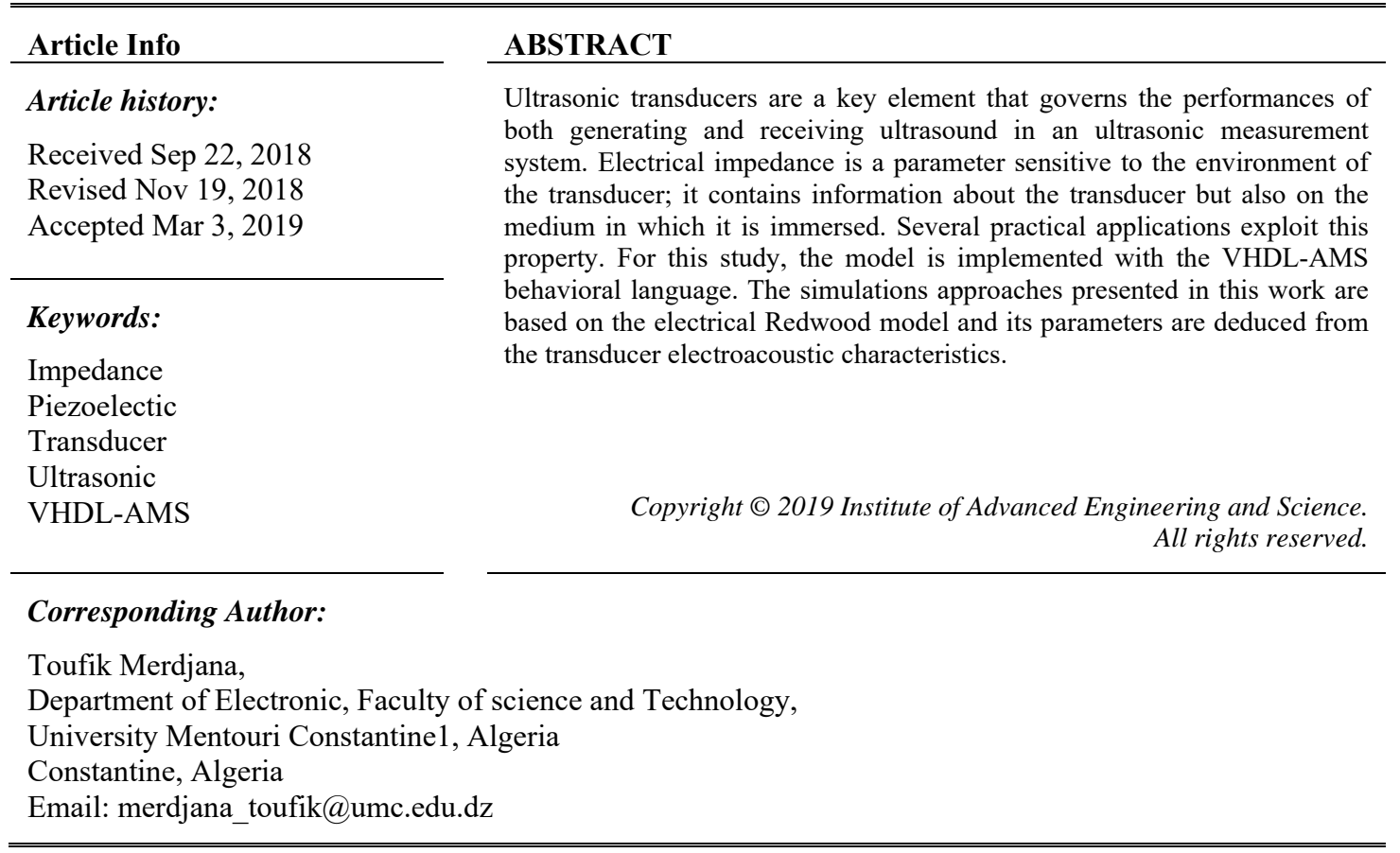

\section{INTRODUCTION}

Ultrasound systems are widely used. They find many applications in engineering, medicine, biology, and other areas. The one indispensible part in these systems is the transducer. These will use the properties of magnetostrictive or piezoelectric materials to convert electrical energy into ultrasonic mechanical energy [1]. Piezoelectric materials have the advantage over other systems of having good performance and being available in very diverse geometries. The electromechanical interaction of piezoelectric transducer, represented by electrical equivalent circuits, was first introduced by Mason [2]. He proposed an exact equivalent circuit that separated the piezoelectric material into an electrical port and two acoustical ports through the use of an ideal electromechanical transformer. The problems with the model are that it required a negative capacitance at the electrical port. Redwood [3] improved this electromechanical model by incorporating a transmission line, making possible to extract useful information on the temporal response of the piezoelectric component.

The Electrical impedance is a parameter sensitive to the environment of the transducer. Several practical applications exploit this property $[4,5]$. The measurement of the impedance makes it possible, for example, to detect the physical or structural modifications of the medium due in particular to damage. This approach is used for non-destructive testing to monitor the condition of structures such as aging and corrosion [6,7]. Frequency analysis of the impedance makes it possible to precisely locate the resonance zone of the transducer. This location can be exploited to control and stabilize the operating frequency of high power systems such as ultrasonic welding devices [8]. Real-time knowledge of the electrical impedance also makes it possible to determine and optimize the power emitted by a transmitter or the sensitivity in reception of a piezoelectric sensor [9]. 
Different approaches were proposed to predict the piezoelectric transducer behavior such as a numerical resolution of piezoelectric equations. Another approach is based on the equivalent electrical circuit simulation using an electric simulator like SPICE (Simulation Program with Integrated Circuit Emphasis) $[10,11]$. However SPICE presents some limitations SPICE is on a continuous basis it cannot support discrete representations, and as a result, it is not suitable for mixed modeling [12, 13]. VHDL-AMS (Very High speed integrated circuit Hardware Description Language Analog and Mixed Signal) is a high-level language that enables numerical and analog simulations, while giving the possibility to simulate systems with different physical magnitudes: mechanical, thermal and electrical. The use of an analysis tool, such as the VHDLAMS behavioral description language, can be a solution to the limitations caused by the use of the SPICE simulator.

\section{THEORY OF PIEZOELECTRIC ULTRASONIC TRANSDUCER}

The piezoelectric material is the principal element in an ultrasonic transducer. The piezoelectric materials have the advantage compared to the other systems of presenting good performances and to be available in very diverse geometries. These materials are generally appeared as a disc, a ring or plate. Our piezoelectric element has the characteristic to vibrate in thickness mode, on only one direction axis ( $\mathrm{z}$ ). This modelling study is thus limited to one geometrical dimension (1-D), with the boundary conditions at the acoustic ports Figure 1 [14].

$$
F=-A T
$$

Where $\mathrm{A}$ is the area of the transducer and $\mathrm{T}$ is the internal stress.
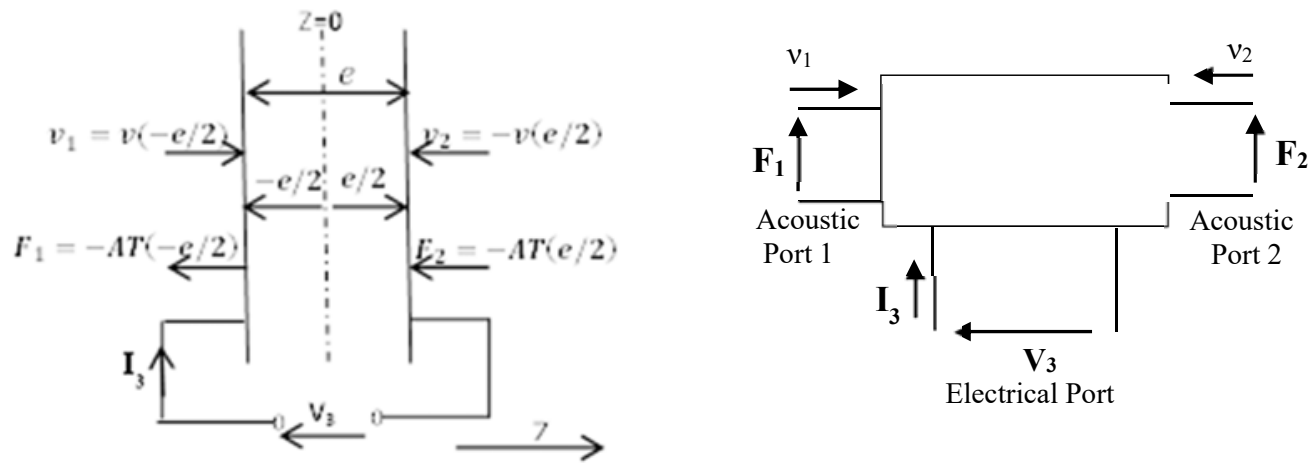

Figure 1. Piezoelectric plate of thickness e and its representation as a three port system

Let's consider e the thickness of the piezoelectric plate and $V_{3}$ is the excitation voltage. $F_{1}$ and $F_{2}$ presented the forces transmitted to the propagation medium on the front and back face of the transducer. $v_{1}$ and $v_{2}$ are the acoustic particles velocities at the front and the back faces of the transducer.

In these conditions, the transfer matrix form (2) which describes the global behavior between the electric excitation port and the two acoustic ports.

$$
\left[\begin{array}{c}
F_{1} \\
F_{2} \\
V_{3}
\end{array}\right]=-j \times\left[\begin{array}{ccc}
Z_{C} / \tan \theta & Z_{C} / \sin \theta & h_{33} / \omega \\
Z_{C} / \sin \theta & Z_{C} / \tan \theta & h_{33} / \omega \\
h_{33 / \omega} & h_{33 / \omega} & 1 / \omega C_{0}
\end{array}\right] \times\left[\begin{array}{c}
v_{1} \\
n_{2} \\
I_{3}
\end{array}\right]
$$

$v_{1}$ and $v_{2}(\mathrm{~m} / \mathrm{s})$ are the acoustic particle velocities at the front and the back faces of the plate, $F_{1}$ and $\mathrm{F}_{2}$ are the acoustic forces at the transducer faces, $\mathrm{Z}_{\mathrm{C}}$ is the acoustic impedance of the piezoelectric material, $\mathrm{h}_{33}=\mathrm{e}_{33} / \varepsilon_{33}$ is the piezoelectricity constant and $\mathrm{C}_{0}$ is the capacitance value of the plate, and $\mathrm{I}_{3}$ is

Study and simulation with VHDL-AMS of the electrical impedance of a piezoelectric ... (Toufik Merdjana) 
the electrical current. $\theta=\omega e \sqrt{\rho / C^{D}}$. The dephasage generates by the propagation with $\omega=2 \pi \mathrm{f}$ is the electrical pulsation where $\mathrm{f}$ is the frequency, $\mathrm{e}$ is the thickness of the piezoelectric plate, $\rho$ is the material density, $C^{D}$ is the elasticity modulus with constant displacement field.

\section{Determination of the electrical impedance of the piezoelectric transducer}

The transducer is assumed here charged to the acoustic ports by homogeneous mediums. We determine the input electrical impedance of the transducer by the impedances of the acoustic loads $Z_{1}$ and $Z_{2}$ respectively acoustic impedance of the front medium and the back medium. At ports acoustic and given the sense of speed in the diagram, we have:

$$
\begin{aligned}
& F_{1}=-Z_{1} v_{1} \\
& F_{2}=-Z_{2} v_{1}
\end{aligned}
$$

We report these relations in the first two equations of the matrix (2) which makes it possible to determine the electrical impedance $\frac{V_{3}}{I_{3}}$

$$
\frac{V_{3}}{I_{3}}=\frac{1}{j C_{0} \omega}\left[1+\frac{C_{0} h_{33}^{2}}{\omega} \frac{-2 Z_{C}[1-\cos \theta]+j\left(Z_{1}+Z_{2}\right) \sin \theta}{\left(Z_{C}^{2}+Z_{1} Z_{2}\right) \sin \theta-j Z_{C}\left(Z_{1}+Z_{2}\right) \cos \theta}\right]
$$

The expression of electrical impedance is generally found in the literature in the following form:

$$
Z_{T}(j \omega)=\frac{1}{j C_{0} \omega}\left[1+\frac{K_{T}^{2}}{\theta} \frac{-2 Z_{C}[1-\cos \theta]+j\left(Z_{1}+Z_{2}\right) \sin \theta}{\left(Z_{C}^{2}+Z_{1} Z_{2}\right) \sin \theta-j Z_{C}\left(Z_{1}+Z_{2}\right) \cos \theta}\right]
$$

Where $\frac{K_{T}^{2}}{\theta}=\frac{C_{0} h_{33}^{2}}{\omega}$ For longitudinal waves, the parameter $K_{T}$ is often defined as the piezoelectric coupling constant for a transversely clamped material; for it is the effective piezoelectric constant used when there is no motion transverse to the electric field.

\section{ELECTRO-ACOUSTIC MODEL OF PIEZOELECTRIC ELEMENT}

For reasons of simplicity, we chose to use Redwood's equivalent electrical circuit. The equivalent circuit of a thickness-mode piezoelectric transducer can be represented by the Redwood model as shown in Figure 2.

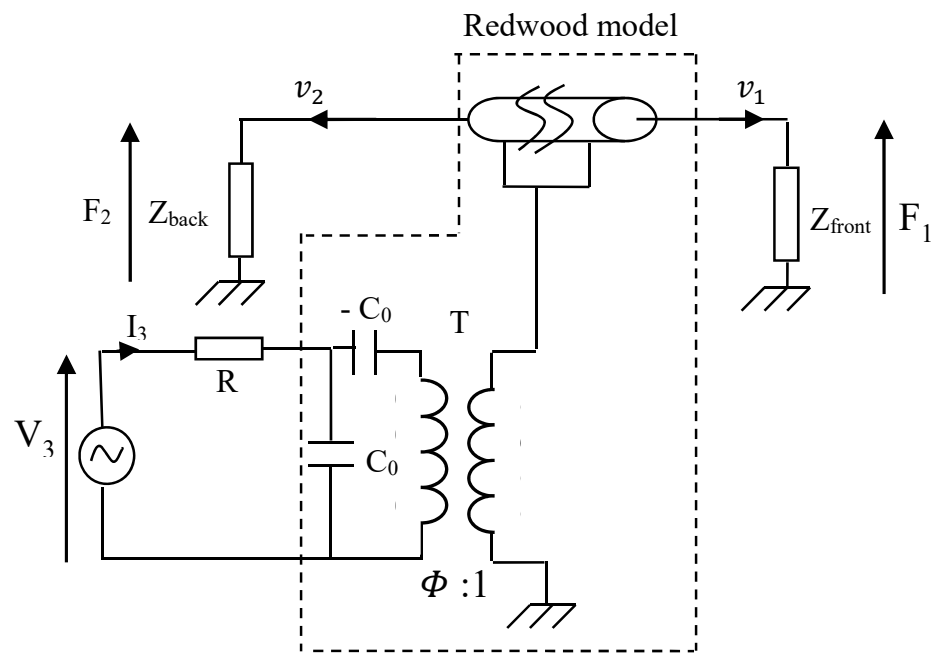

Figure 2. Equivalent circuit of Redwood's model

The model is divided in two parts. The first one is the electrical port which includes the capacitors $\mathrm{C}_{0}$ and $-\mathrm{C}_{0}$ that represent the capacitance motional effect. This electrical port is connected to a resistance $\mathrm{R}$ 
and a voltage source noted $\mathrm{V}_{3}$. The second part is composed by the two acoustic ports, $\mathrm{T}$ is an ideal electroacoustic transformer with a ratioh ${ }_{33} C_{0}$. Piezoceramic layer is assimilated to a propagation line characterized by its characteristic impedance $Z_{0}\left(Z_{0}=Z_{C} \cdot A\right), A$ is the area and the propagation timeTd $\left(T d=e / v^{D}\right)$, with $v^{D}$ is the acoustic velocityand $e$ is the thickness.

One branch of the piezoceramic layer is in contact with the back medium $Z_{\text {back }}$ and the other is in contact with the propagation medium $Z_{\text {front. }}$

\section{VHDL-AMS BEHAVIORAL MODEL OF TRANDUCER}

Before discussing the modeling of transducers in VHDL-AMS, we quickly present this programming language. The VHDL-AMS is an IEEE standard [15]. It was developed as an extension of VHDL to enable the modeling and simulation of circuits and analog and digital - analog mixed systems.

The VHDL-AMS implementation of the previous model as shown in Figure 2 is divided in two parts. First is the declaration of the entity which is composed of the physical characteristics of the transducer and the different terminals used in connection. Each TERMINAL depends of the physical nature of the relation to be implemented to describe the element. Using the statements ELECTRICAL in the electrical domain and KINEMATIC_V in the acoustic domain. The second part of the model is the architecture which establishes the physic laws related to the mathematical relation between each terminal [16].

In this approach, electrical and acoustic elements are described. Electrical element is defined with voltage and current quantities and acoustic element with force and velocity quantities [17, 18]. The VHDL-AMS implementation of Redwood's equivalent model as shown in Figure 2 is given by:

ENTITY Redwood IS

GENERIC $\left(\mathrm{C}_{0}, \mathrm{kt}, \mathrm{Z}_{0}\right.$, Td: real);

PORT (TERMINAL p, m: electrical; TERMINAL t11, m11, t22, m22: kinematic_v);

END ENTITY Redwood;

ARCHITECTURE structure OF Redwood IS

TERMINAL p1: electrical;

TERMINAL $\mathrm{t} 1, \mathrm{t} 1 \mathrm{x}, \mathrm{t} 2 \mathrm{x}$ : kinematic_v;

QUANTITY $v_{1}$ across $i_{1}$ through $\mathrm{p}$ TO m;

QUANTITY $v_{2}$ across $i_{2}$ through $p$ TO $p 1$;

QUANTITY vte across ite through $\mathrm{p} 1 \mathrm{TO} \mathrm{m}$;

QUANTITY pti across uti through t1 TO kinematic_v_ground;

QUANTITY p1xr across $\mathrm{u} 1 \mathrm{xr}$ through $\mathrm{t} 1 \mathrm{x}$ TO $\mathrm{t} 1$;

QUANTITY p $2 x r$ across $\mathrm{u} 2 \mathrm{xr}$ through $\mathrm{t} 2 \mathrm{x} \mathrm{TO} \mathrm{t} 1$;

QUANTITY $\mathrm{p} 1 \mathrm{x}$ across $\mathrm{u} 2 \mathrm{x}$ through $\mathrm{t} 1 \mathrm{x}$ TO $\mathrm{t} 11$;

QUANTITY $\mathrm{p} 2 \mathrm{x}$ across $\mathrm{u} 1 \mathrm{x}$ through $\mathrm{t} 2 \mathrm{x}$ TO $\mathrm{t} 22$;

QUANTITY p11 across $\mathrm{t} 11 \mathrm{TO} \mathrm{t} 1$;

QUANTITY p22 across t22 TO t1;

\section{BEGIN}

$\mathrm{i} 1==\mathrm{C}_{0} * \mathrm{v}_{1}{ }^{\prime} \operatorname{dot}$;

$\mathrm{i} 2==-\mathrm{C}_{0} * \mathrm{v}_{2}{ }^{\prime}$ dot;

$\mathrm{pti}==\mathrm{kt} *$ vte;

$\mathrm{Uti}==-\mathrm{ite} / \mathrm{kt}$;

$\mathrm{p} 1 \mathrm{xr}==\mathrm{p} 22$ 'DELAYED $(\mathrm{Td})-\mathrm{p} 1 \mathrm{x}$;

$\mathrm{p} 2 \mathrm{xr}==\mathrm{p} 11$ 'DELAYED $(\mathrm{Td})-\mathrm{p} 2 \mathrm{x}$;

$\mathrm{p} 1 \mathrm{x}==\left(\mathrm{u} 1 \mathrm{x}+\mathrm{u} 2 \mathrm{x}^{\prime} \text { DELAYED }(\mathrm{Td})\right)^{*} \mathrm{Z}_{0} / 2.0$;

$\mathrm{p} 2 \mathrm{x}==(\mathrm{u} 2 \mathrm{x}+\mathrm{u} 1 \mathrm{x} \text { 'DELAYED }(\mathrm{Td}))^{*} \mathrm{Z}_{0} / 2.0$;

END ARCHITECTURE structure;

\section{SIMULATION RESULTS}

We presentons in this part the results of simulation with VHDL-AMS of the input electrical impedance of the transducer. The studied transducer is built with PZT ceramic of P1-88 type, produced by Quartz and Silice society, vibrating in thickness mode at a frequency of $2.25 \mathrm{MHz}, 1 \mathrm{~mm}$ thick and area $\mathrm{A}=132.73 \mathrm{~mm}^{2}$. These characteristics are recalled in Table 1 .

$$
C_{0}=\varepsilon_{33}^{S} A / e \quad ; \quad v^{D}=\sqrt{\varepsilon_{33}^{S} / \rho} \quad ; \quad K_{t}=h_{33} \sqrt{\varepsilon_{33}^{S} / C_{33}^{D}} \quad ; \quad Z_{c}=\rho v^{D}
$$

Study and simulation with VHDL-AMS of the electrical impedance of a piezoelectric ... (Toufik Merdjana) 
Table 1. Transducer acoustic characteristics [19]

\begin{tabular}{cccc}
\hline Paramètrs & Definition & Value & Unit \\
\hline$\rho$ & Density & 7700 & $\mathrm{Kg} / \mathrm{m}^{3}$ \\
$\mathrm{~V}^{\mathrm{D}}$ & Acoustic velocity & 4530 & $\mathrm{~m} / \mathrm{s}$ \\
$\mathrm{Z}_{0}$ & Acoustic impedance & 34.9 & $\mathrm{Mrayls}$ \\
$\mathrm{C}_{0}$ & Capacitor of the ceramic & 759 & $\mathrm{PF}$ \\
$\mathrm{K}_{\mathrm{t}}$ & coupling factor & 0.49 & - \\
$\mathrm{C}_{33}^{\mathrm{D}}$ & Elastic constant & $15.8 \times 10^{10}$ & $\mathrm{~N} / \mathrm{m}^{2}$ \\
$\varepsilon_{33}^{\mathrm{S}}$ & dielectric constant & $870 \varepsilon_{0}$ & $\mathrm{~F} / \mathrm{m}$ \\
$\mathrm{h}_{33}$ & piezoelectric constant & $1.49 \times 10^{9}$ & - \\
$\tan \delta_{e}$ & Dielectric loss factor & 0.02 & - \\
\hline
\end{tabular}

The frequential transducers response study is essential to predict the sensitivity of the system for the various analyzed mediums. Table 2 gives acoustic characteristics of simulated mediums. The amplitude of the transducer is fixed at 1 Volt with a frequency of $2.25 \mathrm{MHz}$.

Table 2. Acoustic characteristic of different mediums [20]

\begin{tabular}{ccc}
\hline Mediums & Acoustic impedance (Mrayls) & Acoustic velocity (kW) \\
\hline Water & 1.49 & 1469 \\
Ethanol & 0.91 & 1158 \\
Paraffin oil & 1.86 & 1420 \\
\hline
\end{tabular}

\subsection{Study of the electrical impedance of the Redwood model}

The complex impedance of the Redwood model is of the form: $Z_{T}(j w)=R+j X=\left|Z_{T}\right| e^{j \theta}$. We denote $f_{1}$ and $f_{2}$ the frequencies for which the impedance module reaches respectively its minimum and maximum values, the frequencies of resonance fr and antiresonance fa are the values for which the global reactance $X$ of the transducer is zero $(\theta=0)$. The simulation code used to obtain the transducer imput electrical impedance is given by:

ENTITY Impedance_Simulation IS

END Impedance_Simulation;

ARCHITECTURE struct OF Impedance_Simulation IS

TERMINAL $\mathrm{n} 1, \mathrm{n} 2$ : ELECTRICAL;

TERMINAL n3, n4: KINEMATIC_V;

CONSTANT e: real: $=1.0 \mathrm{e}-3$

CONSTANT A: real: $=132.73 \mathrm{e}-3$

CONSTANT $v^{D}:$ real: $=4530.0$

CONSTANT fo: real: $=2.25 \mathrm{e} 6$

CONSTANT $Z_{\mathrm{c}}$ : real:=34.9e6;

CONSTANT kt: real: $=0.49$;

CONSTANT epsi 0 : real: $=8.8542 \mathrm{e}-12$;

CONSTANT epsi $i_{33}:$ real: $=650.0$;

CONSTANT $r_{0}$ : real: $=3300.0$

CONSTANT h: real: $=\mathrm{kt} * v^{D *} \operatorname{sqrt}\left(\mathrm{ro} /\left(\mathrm{epsi}_{0} * \mathrm{epsi}_{33}\right)\right)$

CONSTANT Co: real: $=\mathrm{A}^{*} \mathrm{epsi}_{33} / \mathrm{e}$;

QUANTITY vac: real spectrum 1.0,0.0;

QUANTITY vinput across ie through n1 to electrical_ground;

\section{BEGIN}

Vinput==vac;

R: ENTITY resistanc (bhv) GENERIC MAP (50.0) PORT MAP (n1, n2);

T1: entity Redwood (bhv) generic map (Co, $\left.\mathrm{K}, \mathrm{A} * \mathrm{Z}_{\mathrm{c}}, e / v^{D}\right)$ PORT MAP (n3, kinematic_v_ground, n4, kinematic $\mathrm{v}$ ground, $\mathrm{n} 2$, electrical ground);

Rfront: ENTITY resistanc (bhv) GENERIC MAP (1.5e6*A) PORT MAP (n4, ground);

Rback: ENTITY resistanc (bhv) GENERIC MAP (445.0 A*) PORT MAP (n3, electrical ground);

END ARCHITECTURE struct;

Figure 3 represents different characteristic curves that can be extracted from the impedance $Z_{T}(j w)$. In practice the frequencies $f_{1}$ and $f r$ on the one hand and the frequencies fa and $f_{2}$ on the other hand are very close.

Int J Pow Elec \& Dri Syst, Vol. 10, No. 2, June 2019 : 1064 - 1071 

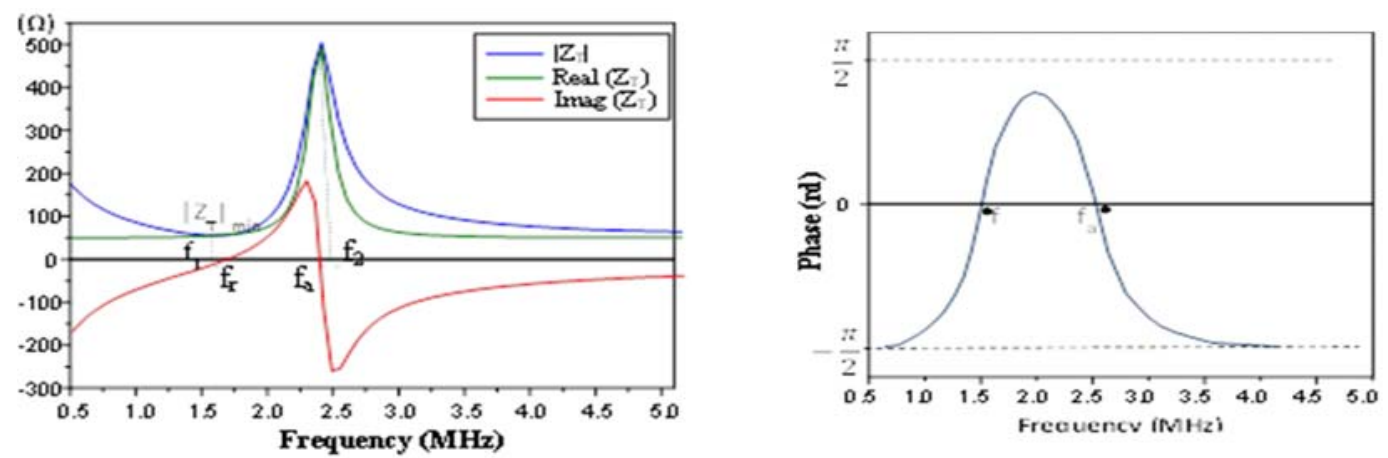

Figure 3. Characteristic curves of the impedance of the Redwood model and Impedance phase

\subsection{Influence of the propagation medium on the impedance modulus}

The vibration modes of the piezoelectric transducers are strongly influenced by the medium in which they are immersed or by the structure of which they are integral. In a homogeneous liquid or gaseous fluid, the corresponding acoustic load modifies the vibration velocity of the transducer and dampens its resonance [21]. Retrodiffused echoes to the transducer generate a reception current. In the case of a thin piezoelectric blade bonded to a solid structure under stress, the influence of the latter is preponderant. These considerations show that the electrical impedance of the transducer is very sensitive to its environment [22]. The transducer is loaded on the front face by three different mediums: water, ethanol and paraffin oil.Their acoustic characteristics are shown in Table 2. The simulation result is shown in the following figure. Figure 4 clearly shows the influence of the medium on the electrical impedance modulus and precisely on $\mathrm{Zmax}$. We note a concordance between the result obtained by VHDL-AMS and the experimental result presented in [23], so a good modeling by VHDL-AMS.
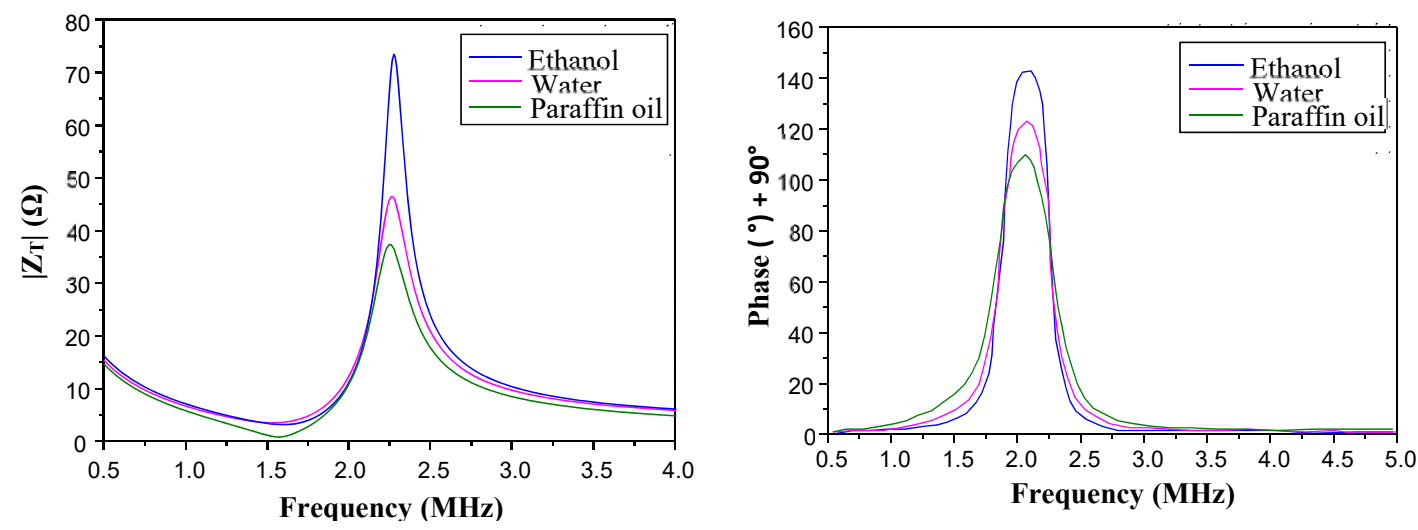

Figure 4. Transducer impedance modulus and the phase for different propagation mediums

\subsection{Influence of coupling factor on the impedance modulus}

From the formula (6) it is found that the electrical input impedance of the transducer is directly related to the coupling factor Kt. The following figure shows the variation of the impedance modulus for different coupling factors. The transducer is loaded on the front face by water as propagation medium.

As shown in Figure 5, the difference between the resonant and antiresonance frequencies is strongly influenced by Kt. The influence of Kt is important on Zmax but negligible on Zmin. 

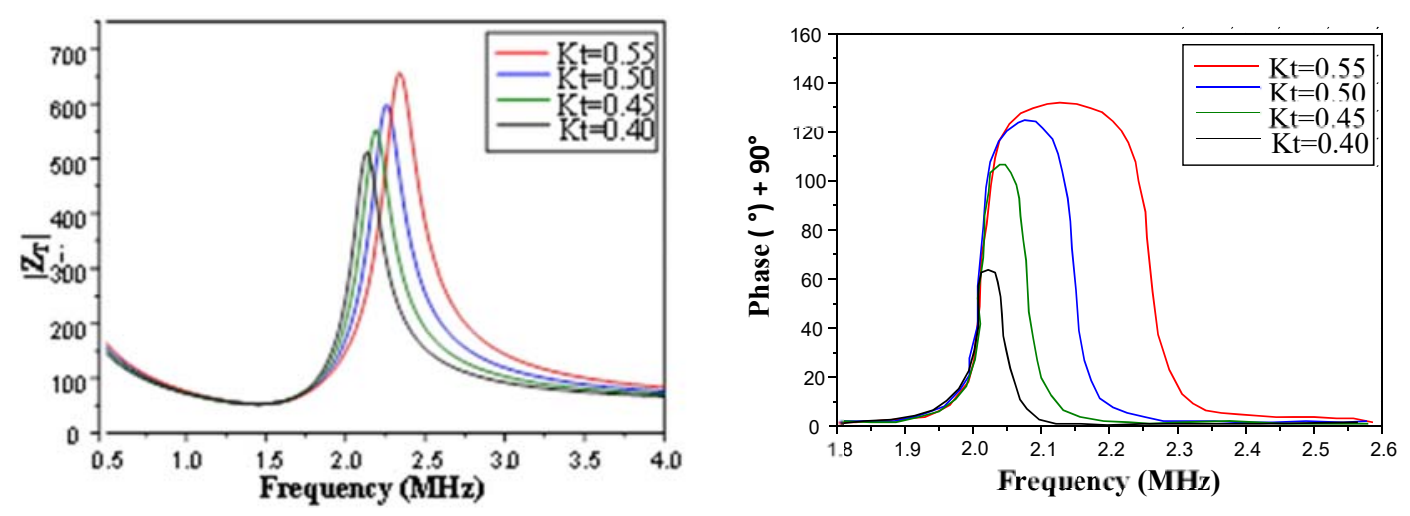

Figure 5. Transducer impedance modulus and phase for different values of $\mathrm{Kt}$

\subsection{Influence of intrinsic dielectric losses on the impedance modulus}

It should be noted that the form (6) of the electrical impedance, does not show the dielectric losses in the ceramic. These can be taken into account when considering a transmission line with losses.

Electrical losses is well known to be the resistance leak of the ceramic capacitance considered as a resistance which depends on the frequency described by relation (7) [24, 25].

$$
R_{e}=\frac{1-K_{t}^{2}}{\omega C_{0} \tan \left(\delta_{e}\right)}
$$

With kt the coupling factor in thickness mode, $C_{0}$ is the capacitance of the ceramic, $\tan \left(\delta_{\mathrm{e}}\right)$ is the losses factor and $\omega$ the pulsation.

Figure 6 shows a growth with $R_{e}$ of the maximum value $Z_{\text {Tmax }}$ of the electrical impedance at the antiresonance frequency, like we can notice a reduction of the phase (the inductive effect decreases in the resonance zone when $R_{e}$ increases). The antiresonance and resonance frequencies as well as the minimum value $Z_{\text {Tmin }}$ are independent of $R_{e}$.
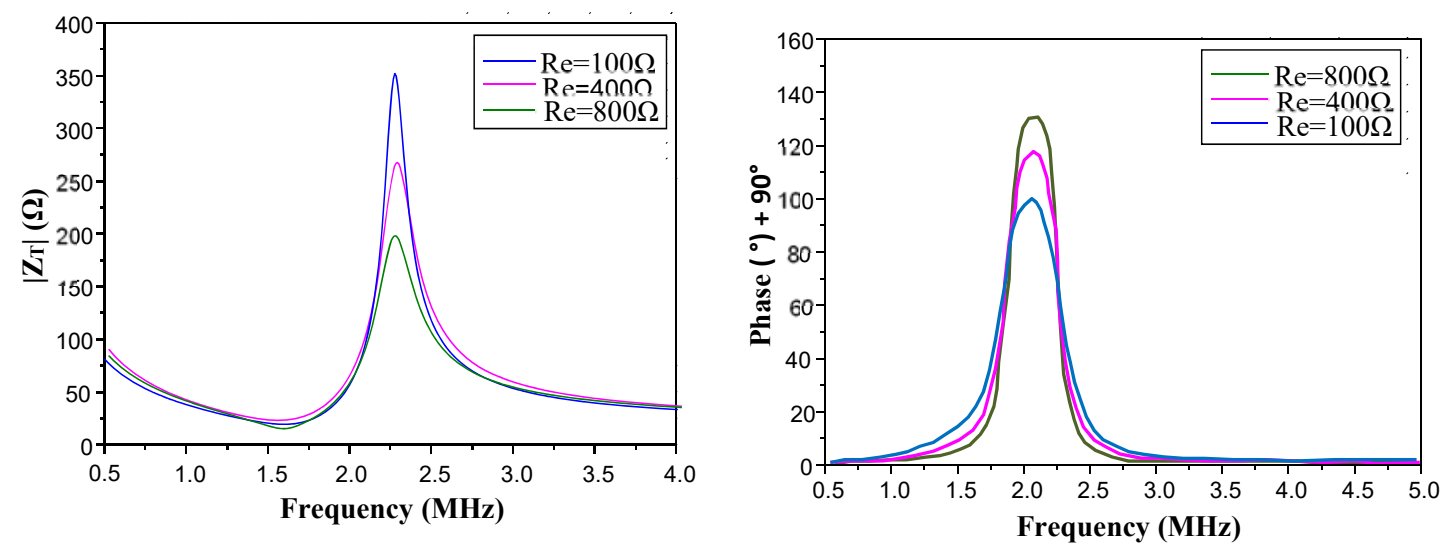

Figure 6. Variations of the impedance modulus and the phase as a function of the dielectric losses

\section{CONCLUSION}

In this paper, a new approach to the ultrasonic transducer modeling system is presented. Our study begins with the presentation of an electric and electroacoustic model of the transducer. From this model we determine the analytical expression of the input electrical impedance. We modeled and simulated the input electrical impedance of the piezoelectric transducer with VHDL-AMS, as well as the influence of the various parameters such as the coupling factor, the propagation medium and the electrical losses on the modulus of the latter. We have shown that the frequency analysis of the electrical impedance can precisely locate the 
resonance zone to control and stabilize the operating frequency of the system. In addition, the use of the VHDL-AMS language has the advantage of combining multi-physical domains, and indicates that the simulation of an ultrasonic detection device, comprising both electronic components and (electromechanical) transducers, is possible with VHDL-AMS. Finally, this study makes it possible to obtain a good representation of the state, the behavior and the performances in real time of the transducer.

\section{REFERENCES}

[1] I. Iswanto, W. S. Agustiningsih, F. Mujaahid, R. Rohmansyah, and A. Budiman "Accumulator Charging Control with Piezoelectric Based on Fuzzy Algorithm Scheduling", TELKOMNIKA Telecommunication, Computing, Electronics and Control, Vol.16, No.2, pp. 635-640, 2018.

[2] W. P. Mason, "Electromechanical Transducers and Wave Filters", New York, D. Van Nostrand, 1942.

[3] R. M. Redwood, "Transcient performance of a piezoelectric transducer", Acoustical Society America, Vol 33, pp. 527-536, 1961

[4] S. R. Ghorayeb, E. Maione and V. La Magna, "Modeling of ultrasonic wave propagation in teeth using PSpice: a comparison with finite element models," in IEEE Transactions on Ultrasonics, Ferroelectrics, and Frequency Control, vol. 48, no. 4, pp. 1124-1131, July 2001.

[5] A.Safari, E. Koray Akdogan "Piezoelectric and Acoustic Materials for Transducer Applications", Springer, Heidelberg, 2008

[6] S. Bhalla and C. K. Soh, "Progress in Structural Health Monitoring and Non- Destructive Evaluation Using PiezoImpedance Transducers", Smart Materials and Structures: New Research, Nova Science Publishers, Inc , New York, pp. 177-228, 2007.

[7] D. M. Peairs, P. A. Tarazaga, and D. J. Inman, "Frequency range selection for impedance-based structural health monitoring", Journal of Vibration and Acoustics, Vol. 129, No. 6, pp. 701-709, 2007.

[8] I. Ibrahim, A. N. Nordin, A. F. M. Mansor, Y. Z. H. Hashim, and I. Voiculescu "Content Cytotoxicity Studies of Colorectal Carcinoma Cells Using Printed Impedance Sensors", Bulletin of Electrical Engineering and Informatics (BEEI) Vol. 6, No. 4, pp. 317 326, 2017.

[9] F. Coutard, "Modélisation et optimisation de structures électroniques pour le conditionnement transducteurs piézoélectriques" PhD. in Electronics, thesis, University of Nancy, France, 2007.

[10] C. G. Hutchens and S. A. Morris, "A Three Port Model for Thickness Mode Transducers Using SPICE II," IEEE 1984 Ultrasonics Symposium, Dallas, Texas, USA, 1984, pp. 897-902.

[11] E. Marione, P. Tortoli, G. Lypacewicz, A. Nowicki, J. M. Reid and L. Fellow "PSPICE modelling of ultrasound transducers" Ultrason., Ferroelectric Frequency Control, vol. 46, No. 2, pp. 399-406, 1999.

[12] S. Jemmali, "Contribution à l'élaboration de méthodologies et d'outils d'aide à la conception de systèmes multitechnologiques" PhD thesis of the National School of Telecommunications of Paris, France, 2003.

[13] T. Merdjana and A. Chaabi "Comparison between VHDL-AMS and PSPICE modeling of ultrasonic transducer for biological mediums" 6th International Conference on Sciences of Electronics, Technologies of Information and Telecommunications (SETIT), Sousse, Tunisia, pp. 183-188, 2012

[14] G. S. Kino, ".Acoustic Waves: Devices, Imaging, and Analog Signal Processing", Ed. Rentice-Hall, Inc. New Jersey. 1987.

[15] IEEE Standard VHDL Analog and Mixed-Signal Extensions, IEEE Std 1076.1-1999, SH94731, IEEE Press, Los Alamitos, 1999

[16] R. Guelaz, D. Kourtiche, M. Nadi and Y. Herve, "Ultrasonic piezoceramic transducer modeling with VHDL-AMS IEEE 1076.1," SENSORS, 2004 IEEE, Vienna, 2004, pp. 87-90 vol.1.

[17] Y. Herve, "VHDL-AMS Applications et enjeux industriels", Paris: Dunod, 2002.

[18] T. Merdjana, A. Chaabi and S. Rouabah "VHDL-AMS and PSPICE modeling of ultrasonic piezoelectric transducer for biological mediums application", Processing - ATSIP', Sousse, Tunisia pp: 523-528, 2014.

[19] Quartz \& Silice. Piezoelectric ceramics (Complete tensor characterization of P188).

[20] Handbook of Chemistry and Physics, 45th edn. Chemical Rubber Co., Cleveland Ohio, 1964.

[21] M. F. A. Shaib, R. A. Rahim, and S.Z.M. Muji, "Development of Non-Invasive Ultrasonic Measuring System for Monitoring Multiphase Flow in Liquid Media within Composite Pipeline", International Journal of Electrical and Computer Engineering (IJECE), Vol. 7, No. 6, pp. 3076-3087, 2017.

[22] T. Merdjana and A. Chaabi "Modelling of PZT/PVDF Ultrasonic Transducer with VHDL-AMS for Medical Applications", Sensors \& Transducers, Vol. 221, Issue 3, pp. 23-29, 2018.

[23] L. Allies, "Ultrasonic Non-Linearity Phenomena Study ", doctoral thesis, Henri Poincaré University, Nancy I, France, 2008.

[24] R. Guelaz and D. Kourtiche. "Double element ultrasonic transducer modelling with VHDL-AMS for lossy piezoceramic", 1st International Conference on Sensing Technology, Palmerston North, New Zealand, 2005.

[25] R. Martinez, A. Vera and L. Leija. "Heat Therapy HIFU Transducer Electrical Impedance Modeling by using FEM". in Proceedings of the conference on Instrumentation Measurement Technology Conference (I2MTC), Montevideo, Uruguay, pp. 299 - 303, 2014. 